\title{
Integración de Pensamiento Computacional en Educación Básica. Dos Experiencias Pedagógicas de Aprendizaje Colaborativo online
}

\section{Integration of Computational Thinking in Compulsory Education. Two Pedagogical Experiences of Collaborative Learning online}

\author{
Xabier Basogain Olabe \\ Universidad del País Vasco / Euskal Herriko Unibertsitatea \\ xabier.basogain@ehu.eus \\ María Elena Olmedo Parco \\ Ministerio de Educación. La Rioja, Argentina \\ melenaolmedoparco@gmail.com
}

\begin{abstract}
Resumen
Los países del mundo han incluido en sus agendas educativas la incorporación del Pensamiento Computacional (PC) en la Educación Básica. A pesar de los esfuerzos de las autoridades escolares, los sistemas escolares tienen dificultades en desarrollar esta propuesta en las aulas tradicionales. En este artículo presentamos dos experiencias basadas en el uso de tecnologías y metodologías educativas que han ayudado a superar estas dificultades, logrando llevar el PC al aula de forma eficaz para los estudiantes, los docentes y centros escolares. Las experiencias se han desarrollado en colaboración con instituciones educativas y ministerios de educación de dos países de Latinoamérica. Las tecnologías educativas utilizadas son los entornos virtuales de aprendizaje (EVA), los sistemas de colaboración síncronos, y los entornos de programación online Scratch/Snap. Las metodologías educativas desarrolladas se basan en el trabajo colaborativo del docente de aula - docente remoto, docentes - estudiantes, y estudiantes - estudiantes. Ambas, tecnologías y metodologías educativas, se han utilizado de forma conjunta para desarrollar a través del PC la capacidad de nuestros estudiantes para resolver problemas relacionados con la vida diaria y con el mundo laboral.
\end{abstract}

Palabras Clave: Pensamiento Computacional, Aprendizaje Colaborativo online, Resolución de Problemas

\begin{abstract}
Countries in the world have included in their educational agendas the incorporation of Computational Thinking (CT) in Compulsory Education. Despite the efforts of the school authorities, the school systems have difficulties in developing this proposal in the traditional classrooms. In this article we present two experiences based on the use of educational technologies and methodologies that have helped overcome these difficulties by bringing the PC to the Classroom effectively for students, teachers and schools. Experiences
\end{abstract}

RED. Revista de Educación a Distancia. Núm. 63, Vol. 20. Artíc. 05, 31-05-2020

DOI: http://dx.doi.org/10.6018/red.409481 
have been developed in collaboration with educational institutions and ministries of education in two Latin American countries. The educational technologies used are virtual learning environments (VLE), synchronous collaboration systems, and Scratch / Snap online programming environments. The educational methodologies developed are based on collaborative work teachers-tutors, teachers-students, and students-students. Both educational technologies and methodologies have been used together to develop, through the PC, the ability of our students to solve problems related to daily life and the working world.

Keywords: Computational Thinking, Online Collaborative Learning, Problem Solving

\section{INTRODUCCIÓN}

Desde inicios de este siglo, en América Latina se viene gestando un profundo proceso de cambio en los Sistemas Educativos a partir de la inclusión de las Tecnologías de la Información y la Comunicación (TIC). Diversos países están realizando importantes inversiones con el propósito de modernizar y mejorar la calidad de la educación que brindan, especialmente, en el nivel primario.

El concepto de Pensamiento Computacional surge en la última década como un tema de especial interés en el ámbito educativo, coincidiendo con el auge de las competencias, capacidades y habilidades que un estudiante debe desarrollar acorde a las exigencias de la Sociedad del Conocimiento. A pesar de que nada está completamente cerrado respecto a su definición urge a los sistemas educativos de nuestra región incorporarlo.

Una de las precursoras del término, Jeannette Wing, en su artículo "Computational Thinking" (Wing, 2006) afirma que el PC es "A way that humans, not computers, think" ("una manera en que los humanos, no las computadoras, piensan). Esto significa que el PC puede ser una buena alternativa para que los estudiantes resuelvan problemas, ya que, como personas, tienen la capacidad de ser creativos, inteligentes y espontáneos. La descripción propuesta por Wing -originalmente usada para explicar el modo de pensar de un científico en computación- ha sido tomada por los estudiosos del campo de la educación para indagar y desarrollar el proceso de enseñanza y aprendizaje mediado por las TIC.

En Uruguay, a partir del Plan Ceibal se han implementado y se impulsan proyectos para fomentar el desarrollo de Pensamiento Computacional en niños en conjunto con el Consejo de Educación de Nivel Primario. Pese a este amplio interés por el concepto, la propuesta de PC continúa en construcción y va evolucionando año a año vinculándola con las competencias que propone la Red Global de Aprendizajes (https://redglobal.edu.uy/) integrando nuevos modos de enseñar y aprender para entender una parte creciente de la realidad del siglo XXI (Gerosa, Koleszar, Carboni, y Gómez, 2019).

Las escuelas participantes de la Red Global de Aprendizaje lo hacen con una propuesta de enseñanza que se mira con una rúbrica hacia dentro, donde lo importante es el proceso, no el resultado. En las clases de PC se aprende, en primer lugar, a cooperar, el estudiante aprende a trabajar con otros logrando resultados en conjunto. En segundo lugar, se aprende con creatividad: el estudiante es capaz de generar nuevas ideas en función a los estímulos recibidos; y en tercer lugar se llega a la resolución de problemas, donde los alumnos buscan resolver problemas de su entorno cotidiano, usando el PC; lo que implica: a) una forma específica de pensarlos; b) poner en juego un conjunto de habilidades prácticas para 
organizar, analizar y representar sus datos; y c) desarrollar y emplear capacidades cognitivas y socio-emocionales (creatividad, pensamiento crítico, carácter, comunicación, colaboración y ciudadanía).

Así, y buscando simplificar el concepto, podemos definir el PC hoy como la capacidad de resolución de problemas del ámbito cotidiano o real, con representación de soluciones mediante algoritmos y buscando que la abstracción sea la protagonista. La idea de que pensar un problema computacionalmente forme a los niños mediante capacidades para vivir en un mundo digitalizado, o que la resolución de problemas implementada pueda trasladarse a otros ámbitos, propone un cambio en relación a aspectos basados solamente en la enseñanza de la programación. La Programación es solo una parte de las Ciencias de la Computación y la propuesta de PC es el modo en que las escuelas se acercan a estas Ciencias.

En este artículo describimos dos experiencias realizadas en países de Latinoamérica que ayudan a explorar las relaciones entre actividades vinculadas al Pensamiento Computacional en las aulas y a sumar experiencias desde la práctica a su construcción.

\section{RESOLUCIÓN DE PROBLEMAS Y PENSAMIENTO COMPUTACIONAL}

En el Currículo de Educación Básica de España se describen las diferentes competencias clave que debe lograr el alumno, y entre ellas está la competencia matemática y competencias básicas en ciencia y tecnología (LOMCE, 2013). La competencia matemática se describe como aplicar el conocimiento matemático para interpretar, describir, explicar y dar respuestas a problemas relacionados con las necesidades de la vida, utilizando modos de pensamiento, representación y herramientas propias del área (Decreto 236/2015, 2016). La competencia matemática está vinculada con una componente práctica: «Aplicar lo que se sabe de cara a resolver una situación». En definitiva, utilizar el saber matemático para resolver problemas y adaptarlo a nuevas situaciones. En los contenidos de esta materia, además de números y operaciones, medida, geometría, tratamiento de la información, azar y probabilidad, está el bloque Resolución de Problemas. Este bloque está orientado a «identificar problemas de la vida cotidiana, reconocer los datos y relaciones relevantes, formular conjeturas, desarrollar estrategias de resolución exacta o aproximada, comprobar conjeturas y resultados, organizar y comunicar los resultados».

Un análisis detallado de los tipos de problemas matemáticos que realizan en la escuela los estudiantes de todo el mundo revela que los problemas comparten una misma estructura. Los problemas incluidos en las pruebas estandarizadas, como las del Programme for International Student Assessment (PISA), son buenos recursos para investigar esta estructura. Nosotros vamos a denominar a estos problemas: problemas Tipo-A (Olabe, Basogain, Olabe, Maíz \& Castaño, 2014). Un ejemplo de problema de la prueba PISA es el siguiente:

"Un ático tiene una forma cuadrada de 12 metros de lado. ¿Cuál es el área del suelo del ático?"

Las tres fases para resolver estos problemas son: correspondencia, identificación de reglas, y aplicación de reglas. La fase de correspondencia consiste en encontrar los datos relevantes del problema, qué datos son relevantes y qué se pide. En este ejemplo es importante saber que la planta tiene una forma cuadrada y que su lado es de 12 metros de

Integración de Pensamiento Computacional en Educación Básica. Dos Experiencias Pedagógicas de Aprendizaje Colaborativo online. Xabier Basogain y María Elena Olmedo

Página 3 de 21 
largo. También es importante tener en cuenta que nos piden calcular el área del suelo. Estos tres datos (cuadrado, 12, área) son el resultado de la fase de correspondencia. La identificación de las reglas es el proceso de determinar qué reglas relacionan los datos que conocemos con los datos que no conocemos. En este caso tenemos que calcular el área, y sabemos que la forma es un cuadrado, por lo tanto, sólo necesitamos una regla, y la regla es la relación entre el área y el lado de un cuadrado. Entonces, La regla es Área = lado * lado. Y finalmente, la aplicación de las reglas consiste en sustituir los datos conocidos en la regla o reglas y determinar la solución del problema. Área = lado * lado; Área =12 * 12; Área $=144$. La solución de un problema Tipo-A es un número, y que generalmente no le dice nada al alumno.

Este modelo de problema Tipo-A no aborda otros problemas más importantes y frecuentes que denominamos problemas Tipo-B. Este tipo de problemas son los que normalmente encontramos en la vida cotidiana, y responden a un paradigma muy diferente. Los problemas Tipo-B tienen características muy diferentes a las características de los problemas Tipo-A: no son deterministas en su solución (la solución no es conocida a priori y no es única); y no son deterministas en el proceso de resolución (el proceso hacia la solución no es conocido a priori, ni es único para las múltiples soluciones).

Un ejemplo de problema Tipo-B que muestra las diferencias con los problemas Tipo-A es el siguiente problema:

"Crea el juego de Pong. La pelota rebota en las paredes y la paleta está controlada por el jugador. Un marcador lleva la cuenta del número de veces que el jugador ha golpeado la bola. Finalmente, si la paleta no logra golpear la pelota y ésta llega a la línea roja del fondo el juego termina." (Basogain, Olabe \& Olabe, 2015).

Este problema tiene múltiples soluciones. El cálculo del área de un cuadrado, un problema Tipo-A, tiene una única solución, y esa solución es conocida antes de que el estudiante resuelva el problema. En el caso del problema del juego de Pong, un problema Tipo-B, existen infinidad de soluciones, y la mayoría de ellas son todavía desconocidas. Un problema Tipo-B no es determinista ni en su solución ni en el proceso de búsqueda de la solución. Además, son iterativos por naturaleza lo que requiere experimentación para su resolución. Su solución es un proyecto donde el alumno sabe si ha logrado el resultado esperado.

\section{El Pensamiento Computacional como instrumento del desarrollo de capacidades}

La propuesta de Plan Ceibal ${ }^{1}$ de impartir clases de Pensamiento Computacional (PC) mediante el Aprendizaje Colaborativo Online en escuelas de nivel primario en Uruguay, tiene como objetivo desarrollar competencias que tienen que ver no solo con lo cognitivo, sino también con lo emocional y la construcción de ciudadanía. Se entiende que, además de promover el desarrollo de la competencia matemática, es una oportunidad para el desarrollo del juicio crítico, el respeto, la tolerancia, el trabajo colaborativo, la autoestima positiva, la creatividad, entre otras.

El Aprendizaje Colaborativo online se desarrolla a través de video conferencia con sesiones dirigidas por un Docente Remoto (DR) en colaboración y coordinación con el

\footnotetext{
${ }^{1}$ El Plan Ceibal de Uruguay se creó en 2007 como un plan de inclusión e igualdad de oportunidades con el objetivo de apoyar con tecnología las políticas educativas uruguayas. Desde su implementación, cada niño que ingresa al sistema educativo público en todo el país accede a una computadora para su uso personal con conexión a Internet gratuita desde el centro educativo.
}

Integración de Pensamiento Computacional en Educación Básica. Dos Experiencias Pedagógicas de Aprendizaje Colaborativo online. Xabier Basogain y María Elena Olmedo

Página 4 de 21 
Docente de Aula (DA). El DR planifica las clases teniendo en cuenta tanto los contenidos específicos para el nivel de los alumnos y de las características del grupo-aula, como las capacidades que propone la Red Global de Aprendizajes.

Dicha Red Global de Aprendizajes es una iniciativa de colaboración internacional que busca integrar nuevas formas de enseñar y aprender en instituciones educativas de diferentes países, a través de un marco común de acciones e investigación. El objetivo es impulsar, sistematizar y evaluar un conjunto de prácticas educativas que tiendan al "aprendizaje profundo" y al desarrollo de "competencias transversales" para la vida. La Alianza Global o NPDL (New Pedagogies for Deep Learning) fue propuesta y convocada por el pedagogo Michael Fullan (Fullan \& Langworthy, 2013). La Administración Nacional de Educación Pública de Uruguay (ANEP) y Plan Ceibal fueron invitados a participar junto con otros seis países: Australia, Canadá, Estados Unidos, Finlandia, Holanda y Nueva Zelanda.

En la Red Global de Aprendizajes la tecnología tiene un rol central como forma de ampliar significativamente el acceso a la información y acelerar los resultados pedagógicos. La Red no proporciona a los docentes un modelo de enseñanza, sino una metodología de análisis y evaluación de las prácticas que cada institución desarrolla, para que puedan mejorarlas, ampliarlas y enriquecerlas con su propia reflexión.

En Uruguay, la Red está integrada por centros educativos de todos los subsistemas de la ANEP: Primaria, Secundaria, Universidad de Trabajo del Uruguay (UTU) y Formación Docente.

La Red Global se basa en el entendimiento de que ningún país, sector u organización tiene todas las respuestas. Más bien, se trata del desarrollo de capacidades en forma colectiva para determinar, aplicar y medir enfoques nuevos e innovadores para el aprendizaje.

En el marco de la propuesta de clases de Pensamiento Computacional (PC), la innovación es la incorporación de las tecnologías en la cual, específicamente la programación de los ordenadores con lenguajes de programación visual, es una ayuda para resolver problemas y situaciones problemáticas.

Como punto de partida, es importante advertir que el surgimiento de una situación problemática en el contexto escolar no aparece por el simple hecho de que el docente la presente a los alumnos y se las plantee como tal. Hace falta que el niño -con la mediación de quien enseña- se acerque a ese problema, lo conozca, lo entienda; es decir, necesita abordarlo para poder comprenderlo, interiorizarlo, asumirlo. Sólo así tendrá sentido pensar en alternativas de solución a esa situación problemática (proceso de resolución).

"Proponer esporádicamente a los estudiantes que resuelvan situaciones problemáticas no es suficiente, ya que crear condiciones favorables para el aprendizaje de esta capacidad demanda construir progresiones, de manera tal de permitir a los estudiantes un desarrollo también progresivo de la capacidad y que puedan analizar cuánto más saben ahora sobre resolver situaciones problemáticas de lo que sabían antes. Labarrere (en Sigarretay Laborde Chacón, 2004) sostiene: La solución de un problema no debe verse como un momento final, sino como todo un complejo proceso de búsqueda, encuentros, avances y retrocesos en el trabajo mental. Este complejo proceso de trabajo mental se materializa en el análisis de la situación ante la cual uno se halla: en la elaboración de hipótesis y la formulación de

Integración de Pensamiento Computacional en Educación Básica. Dos Experiencias Pedagógicas de Aprendizaje Colaborativo online. Xabier Basogain y María Elena Olmedo

Página 5 de 21 
conjeturas; en el descubrimiento y selección de posibilidades; en la previsión y puesta en práctica de procedimientos de solución". ${ }^{2}$

\section{Problema Situado y PC}

¿A qué nos referimos cuando hablamos de un Problema Situado (PS)? Para responder vamos a situarnos en la concepción de Pensamiento Computacional en los siguientes términos:

- El Pensamiento Computacional es un proceso de pensamiento, independiente de la tecnología.

- El Pensamiento Computacional es un tipo específico de resolución de problemas que implica capacidades distintas, por ejemplo, ser capaz de diseñar soluciones para ser ejecutadas por un ordenador, un humano, o una combinación de ambos.

- El Pensamiento Computacional es un proceso de resolución de problemas incluye las siguientes características, sin estar limitado por ellas:

1) Formular problemas de una manera que nos permita usar un ordenador y otras herramientas para ayudar a resolverlos.

2) Organizar y analizar datos de una manera lógica.

3) Representar datos a través de abstracciones tales como modelos y simulaciones.

4) Automatizar soluciones mediante el pensamiento algorítmico (una serie de pasos ordenados).

5) Identificar, analizar e implementar posibles soluciones con el objetivo de conseguir la combinación más eficaz de pasos y recursos.

6) Generalizar y transferir este proceso de resolución de problemas a una amplia variedad de problemas.

Se promueve el desarrollo de habilidades de Pensamiento Computacional en el alumnado para que puedan pensar de manera diferente, expresarse a través de una variedad de medios, resolver problemas del mundo real y analizar temas cotidianos desde una perspectiva diferente. Entonces, hablamos de problemas del mundo real, "situados", porque pueden ser de la realidad de la escuela o de la comunidad en que está inserta, de la cotidianeidad del niño, es decir que lo vive en el día a día, auténticos. La tabla 1 muestra tres de estos problemas identificados por los alumnos en las escuelas de Uruguay que se encuentran en diferente estadio de desarrollo (CREA2, 2018).

Tabla 1.- Ejemplos de Problema Situado desarrollados en escuelas de Uruguay.

\begin{tabular}{|c|c|c|}
\hline Ejemplo 1 (Sexto curso) & Ejemplo 2 (Quinto curso) & Ejemplo 3 (Sexto curso) \\
\hline $\begin{array}{l}\text { Sexto año realizó una asamblea } \\
\text { buscando un problema que afecta a } \\
\text { nuestra escuela. Es así que el } \\
\text { problema detectado está a la entrada } \\
\text { de nuestra escuela. Tiene siete } \\
\text { escalones para ascender y personas } \\
\text { con alguna discapacidad motora no } \\
\text { pueden subir por sí mismos. } \\
\text { Posibles soluciones: } \\
\text { - Colocar rampa. }\end{array}$ & $\begin{array}{l}\text { En la escuela y en su entorno } \\
\text { hay basura. } \\
\text { ¿Qué se pretende? } \\
\text { Desde el curso: } \\
\text { A. Dentro del proceso } \\
\text { reciclado del papel, crear un } \\
\text { dispositivo que sirva para }\end{array}$ & $\begin{array}{l}\text { Este proyecto retoma la } \\
\text { indagación sobre el perfil acústico } \\
\text { de la institución escolar. } \\
\text { Motivados por la necesidad de } \\
\text { obtener un cambio con respecto al } \\
\text { sonido en diferentes ámbitos de la } \\
\text { escuela, se valoran los hábitos y } \\
\text { conciencia sobre el daño que } \\
\text { producen los ruidos molestos y la }\end{array}$ \\
\hline
\end{tabular}

${ }^{2}$ Abordaje y resolución de situaciones problemáticas- Documento de Acompañamiento № 13. Programa Nuestra Escuela. Ministerio de Educación de la República Argentina (2017).

Integración de Pensamiento Computacional en Educación Básica. Dos Experiencias Pedagógicas de Aprendizaje Colaborativo online. Xabier Basogain y María Elena Olmedo

Página 6 de 21 


\begin{tabular}{|c|c|c|}
\hline $\begin{array}{l}\text { - Colocar ascensor. } \\
\text { - Insertar baranda. } \\
\text { • Colocar escalera mecánica. } \\
\text { Este problema se puede complejizar, } \\
\text { ver las diferentes opciones, visualizar } \\
\text { videos que ofrezcan propuestas } \\
\text { diferentes a las cotidianas, ver } \\
\text { viabilidad, inclinación de la rampa, la } \\
\text { relación inclinación- longitud de la } \\
\text { misma, trabajar con contenidos de } \\
\text { física, materiales más convenientes, } \\
\text { características de los mismos, etc. Por } \\
\text { último, elegir cada grupo de clase una } \\
\text { solución y prototiparlos. }\end{array}$ & $\begin{array}{l}\text { mezclar la pasta luego de } \\
\text { poner el papel en remojo. } \\
\text { B. Crear un dispositivo que en } \\
\text { el momento que se coloque la } \\
\text { basura en el tacho escriba un } \\
\text { mensaje. }\end{array}$ & $\begin{array}{l}\text { responsabilidad de cada miembro } \\
\text { como agente de cambio. } \\
\text { Para materializar este objetivo, los } \\
\text { alumnos deciden la programación } \\
\text { de un instrumento que mida y } \\
\text { alerte de los sonidos fuertes en el } \\
\text { entorno utilizando Scratch. Para } \\
\text { ello se propone la construcción de } \\
\text { un dispositivo que funcione con } \\
\text { señales lumínicas y que alerte } \\
\text { sobre los niveles de alta } \\
\text { contaminación acústica, en los } \\
\text { espacios compartidos. }\end{array}$ \\
\hline
\end{tabular}

La relación entre la programación y el problema situado es directa a la hora de explorar el modo en que se aborda y resuelve un problema. La programación se utiliza como guía para pensar cómo atender un problema desde pensamiento computacional (involucre o no su resolución la tecnología). Se busca enseñar que resolver un problema implica un conjunto de acciones: a) definirlo, b) elaborar una estrategia de solución posible: descomponer en subproblemas, c) lograr diferenciar lo relevante de lo que no lo es, d) abstraer, e) definir un conjunto ordenado de pasos para alcanzar el objetivo, f) formular la estrategia de solución de un modo comunicable y legible para terceros, g) ejecutar la estrategia, y h) evaluar posibles mejoras en términos de eficiencia de tiempos, cantidad de pasos, legibilidad.

La resolución de este tipo situación-problema o de los problemas Tipo-B requiere de un lenguaje diferente del lenguaje algebraico de las matemáticas que se enseña en el aula de forma tradicional. Se requiere un lenguaje generativo que permita crear, simular, explorar, comprobar y experimentar la resolución de los problemas.

Los desarrollos realizados en las últimas décadas en ingeniería informática ofrecen nuevos paradigmas. En particular, los paradigmas basados en lenguajes y objetos han revolucionado la forma en la que la mente aborda las tareas de pensar, crear y resolver problemas. Los entornos gráficos de programación como Alice, Greenfoot, y Scratch (Utting, Cooper, Kolling, Maloney \& Resnick, 2010) están diseñados para introducir y desarrollar conceptos de computación y programación basándose en las ideas fundamentales de los lenguajes orientados a objetos.

Estos entornos de programación están orientados para niños y jóvenes. Son aplicaciones interactivas donde los estudiantes pueden experimentar y explorar realizando programas en entornos gráficos. Estos programas gráficos proveen entornos visuales manipulables donde el alumnado ve en la pantalla del ordenador sus instrucciones y acciones de una forma sencilla y directa.

En estos entornos, las acciones ocurren entre entidades llamadas objetos, que están caracterizados por sus estados, sus comportamientos, y sus interacciones con otros objetos. La unión conceptual de objeto y su representación gráfica es la que dota a estos entornos de programación con un valor pedagógico óptimo.

Scratch y Snap (Maloney, Resnick, Rusk, Silverman \& Eastmond, 2010; Scratch, 2019; Snap, 2019) son dos entornos gráficos de programación que permiten una fácil recreación donde podemos experimentar con los procesos de resolución de problemas Tipo-B.

Integración de Pensamiento Computacional en Educación Básica. Dos Experiencias Pedagógicas de Aprendizaje Colaborativo online. Xabier Basogain y María Elena Olmedo

Página 7 de 21 
La figura 1 muestra la resolución del problema del juego de Pong ${ }^{3}$ mejorado respecto al enunciado original (dos jugadores golpean la pelota con sus palas, el juego incluye marcadores, cronómetro y sonidos) en el entorno de programación Scratch 3.0.

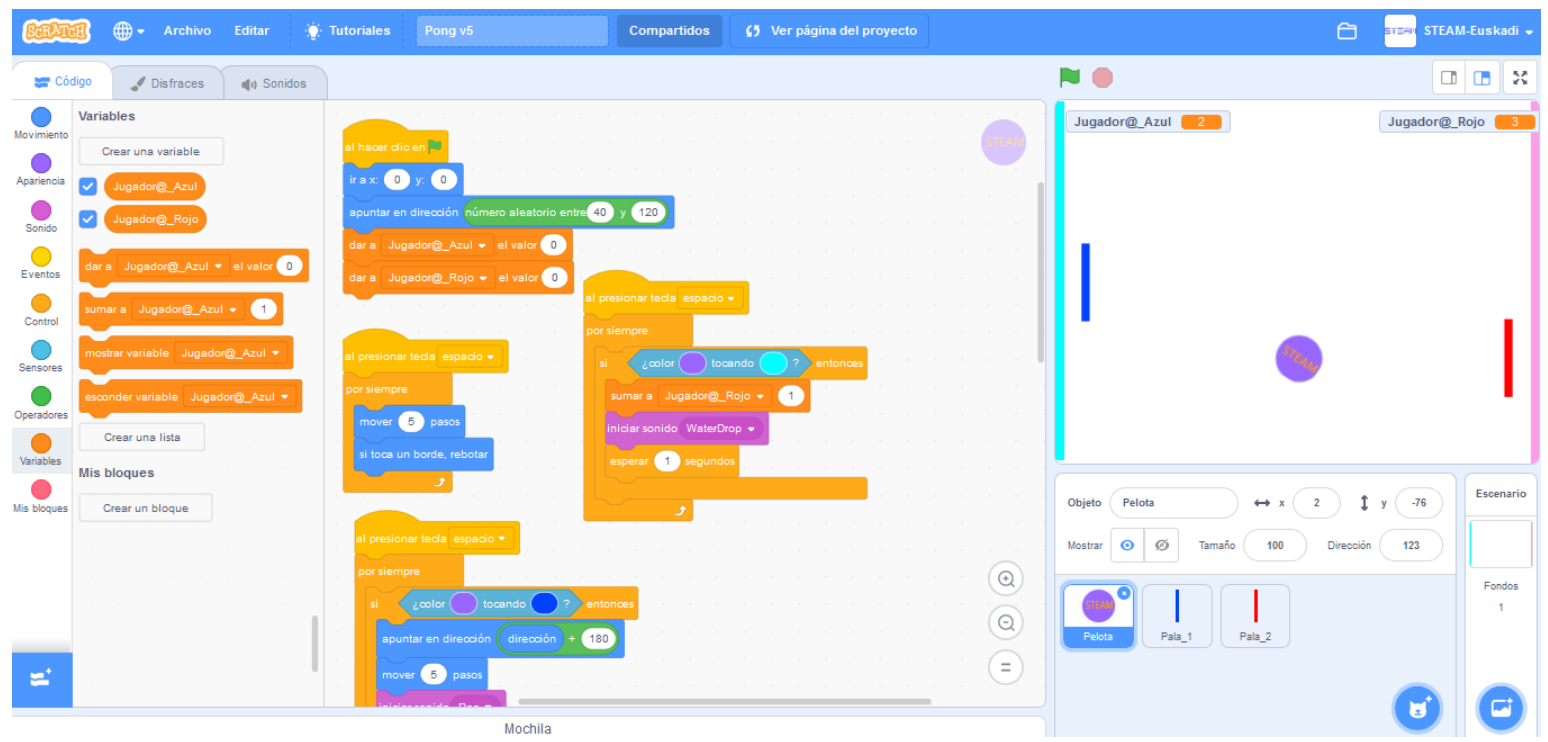

Figura 1.- Resolución del problema Juego de Pong en el entorno de programación Scratch 3.0

\section{APRENDIZAJE COLABORATIVO ONLINE DE PENSAMIENTO COMPUTACIONAL}

El ambiente colaborativo al que hacemos referencia en la Introducción se ha hecho realidad en dos experiencias pedagógicas escolares: 1) Proyecto PC en Uruguay (Pensamiento Computacional por Docentes Remotos), y 2) Proyecto PC en República Dominicana (Curso PC-01).

\section{Proyecto Pensamiento Computacional por Docentes Remotos}

El equipo de Laboratorios digitales del Plan Ceibal, conjuntamente con la Fundación Sadosky de Argentina, lleva adelante el Programa Pensamiento Computacional en aquellas escuelas uruguayas inscriptas al Programa en forma voluntaria. Este programa se hace concreto en una iniciativa llamada "Docentes Remotos" que permite a alumnos de escuelas primarias "asistir" a clases en las cuales el Aprendizaje Colaborativo Online se lleva a cabo a través de video conferencias dictadas por docentes desde la Argentina (CREA2, 2018).

Como ya se ha dicho, en estas clases el eje troncal es el Problema Situado identificado por los alumnos. El proceso para identificarlo, definirlo, llegar a soluciones alternativas de ese problema, será el hilo conductor de cada clase de Pensamiento Computacional. En este marco, la programación es una ayuda para resolver problemas y, como tal, está al servicio del problema situado.

\footnotetext{
${ }^{3}$ https://scratch.mit.edu/studios/18382389/

Integración de Pensamiento Computacional en Educación Básica. Dos Experiencias Pedagógicas de Aprendizaje Colaborativo online. Xabier Basogain y María Elena Olmedo

Página 8 de 21
} 
El resultado es la exploración del modo en que se aborda y resuelve un problema y el uso de la programación como guía para pensar cómo atender un problema desde Pensamiento Computacional (involucre o no su resolución la tecnología).

Naturalmente, es necesario que el Docente Remoto (DR) y el Docente de Aula (DA) tengan conocimiento acabado del guion a trabajar: objetivos, propósitos, actividades a realizar, aplicaciones a usar, y recursos necesarios. El conocimiento del contenido a enseñar y de la intencionalidad didáctica de cada actividad es imprescindibles para el éxito de la clase.

El principal aspecto a considerar es el grupo con el que se establece la comunicación. El conocimiento de la situación socioeconómica de los niños de la escuela, por ejemplo, se puede ir mejorando a través del diálogo que se establece en coordinación con el Docente de Aula. La clave para la motivación de los niños es que sean ellos los protagonistas, el trabajo en equipo y que, al pensar en un problema para aplicar el Pensamiento Computacional, sea uno de su propia realidad: pensar sobre situaciones de su entorno.

La planificación de la clase por Videoconferencia que dura 45 minutos supone tener claro qué rol asumirá cada uno en cada actividad del guion: estimar los tiempos que requerirán considerando las necesidades del grupo, pensar los acentos en la interacción, la organización del trabajo -dupla o individual- y el uso o no de las computadoras de los niños.

Otros asuntos prácticos son - algunos obvios - el saludo inicial y de despedida, el contacto visual y/o verbal con el maestro, la promoción de un clima adecuado de clase en el que tanto los niños como el DA y el DR interactúen y realicen las actividades previstas. La construcción de la pareja pedagógica es paulatina y se basa en la empatía y la confianza desarrollada durante la Coordinación y la Videoconferencia.

Los primeros guiones son algo más estructurados pero a medida que se avanza en el proyecto y los niños van adquiriendo más seguridad, los Docentes Remotos van transitando hacia un rol de guía activo: pregunta o responde, orienta, anima. Igualmente nunca está inactivo. El tono de voz es también importante. No son conferencias magistrales pero es necesario que todos lo oigan y que también haga las inflexiones necesarias para evitar una exposición monocorde, tal como en un diálogo natural. La figura 2 muestra una clase por Videoconferencia en cuarto año de una escuela en Uruguay.
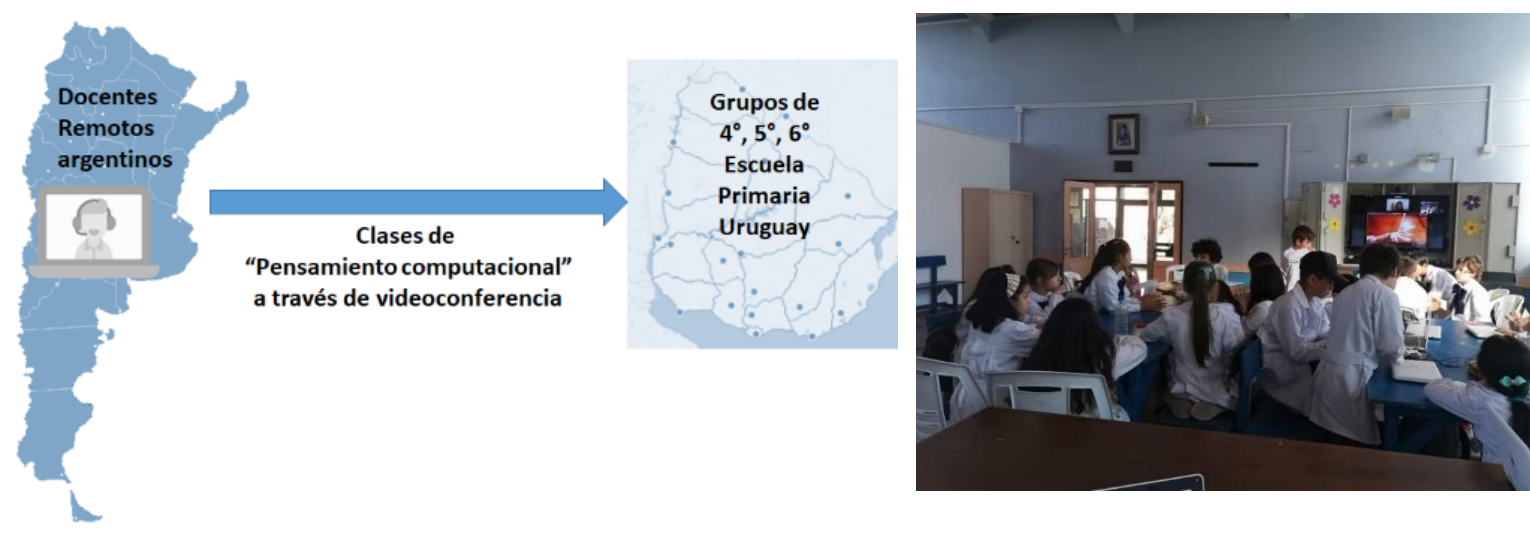

Figura 2.- Proyecto PC con Docente Remoto y, una sesión de Videoconferencia de clase de PC con la docente remota María Elena Olmedo Parco.

Integración de Pensamiento Computacional en Educación Básica. Dos Experiencias Pedagógicas de Aprendizaje Colaborativo online. Xabier Basogain y María Elena Olmedo

Página 9 de 21 
El objetivo del DR es llegar con las clases de PC a la construcción de un prototipo de dispositivo tecnológico vinculado a la resolución del problema con los materiales disponibles en la escuela. Plan Ceibal distribuye una caja con materiales para aprendizaje con material concreto y la Placa BBC Micro Bit para cada alumno de sexto año. La solución concreta y final del problema en general requiere de una gestión institucional (del Director y DA) que excede al DR. Dependerá de las posibilidades de cada escuela la gestión de la solución concreta del problema elegido por los alumnos.

Ya sea que se pueda o no se pueda construir el prototipo automatizado, es muy importante el proceso de la meta cognición. Recordando las competencias de la Red Global, la meta cognición permite a los estudiantes tomar conciencia sobre su propio proceso de aprendizaje. Sin esa reflexión, los alumnos no logran el aprendizaje profundo. No pueden quedarse con la idea de "haber jugado" con Scratch. El desarrollo de la meta cognición es uno de los objetivos para las clases de PC, reflexionar sobre la propia conciencia del pensamiento y del propio proceso de aprendizaje para aplicar a diferentes situaciones de la vida cotidiana. La tabla 2 recoge las preguntas sobre su propio pensamiento que se corresponden con distintos usos.

Tabla 2.- Meta cognición de las clases de PC realizadas por Docente Remoto.

\begin{tabular}{|c|c|c|c|}
\hline $\mathbf{n}^{\mathbf{0}}$ & Pregunta & Descripción & Ejemplo \\
\hline 1 & ¿Qué he aprendido? & $\begin{array}{c}\text { Uso tácito del pensamiento. El alumno debe } \\
\text { reflexionar sobre su propio pensamiento. }\end{array}$ & $\begin{array}{c}\text { ¿Cómo identifiqué el } \\
\text { problema? }\end{array}$ \\
\hline 2 & ¿Qué pasos he seguido? & $\begin{array}{c}\text { Uso consciente. Aquí se pretende que el } \\
\text { estudiante recuerde lo que ha hecho para } \\
\text { producir el aprendizaje }\end{array}$ & $\begin{array}{c}\text { ¿El problema se ha } \\
\text { divido en partes? }\end{array}$ \\
\hline 3 & ¿Para qué me ha servido? & $\begin{array}{c}\text { Reflexión sobre el uso estratégico, la utilidad } \\
\text { de lo aprendido. Esto es fundamental para } \\
\text { tomar conciencia de que va creciendo el } \\
\text { conocimiento }\end{array}$ & $\begin{array}{c}\text { ¿En qué información } \\
\text { importante me apoyé } \\
\text { para dar solución al } \\
\text { problema? }\end{array}$ \\
\hline 4 & $\begin{array}{c}\text { ¿En qué otras situaciones } \\
\text { puedo utilizarlo? }\end{array}$ & Uso reflexivo de lo aprendido. & $\begin{array}{c}\text { ¿Qué serie de pasos } \\
\text { seguí para resolverlo? }\end{array}$ \\
\hline
\end{tabular}

\section{Proyecto Curso PC-01}

En este proyecto presentamos un curso introductorio de Pensamiento Computacional para educación básica donde se enseñan los "conceptos" y "procesos" básicos de computación para jóvenes estudiantes de 10-12 años (College Board, 2016).

El curso está diseñado para ser impartido en formato híbrido o mixto de educación. El maestro está presente con los estudiantes en el aula y utiliza una plataforma de aprendizaje en línea (Moodle) en la que se ha dispuesto el curso PC-01 que incluye videotutoriales, prácticas, auto-tests, tests, y tareas evaluadas por los compañeros (Basogain, Olabe, Olabe \& Rico, 2018; Basogain et al., 2016).

Los docentes fomentan la colaboración y la comunicación en el aula, y pueden personalizar y diferenciar el progreso en el aula. Los estudiantes también se benefician de la plataforma de aprendizaje al aprender a trabajar con sus compañeros en proyectos, y al desarrollar habilidades de colaboración y resolución de problemas.

En estas clases, el eje troncal es el diseño y realización de un proyecto Scratch que resuelve un Problema Tipo-B. Denominamos Tarea Evaluada por Compañeros (TEC) a la 
tarea realizada por el alumno para resolver un problema mediante la creación de un proyecto Scratch. Los proyectos son evaluados por compañeros estudiantes utilizando una rúbrica común. En inglés, el término P2P (peer to peer) se utiliza para identificar este tipo de evaluación por pares, que en Moodle se implementa con la actividad denominada Taller.

Los docentes disponen de una serie de herramientas de aprendizaje analítico integrado en Moodle que les permiten implementar evaluaciones del progreso de los estudiantes, y evaluaciones colectivas e individuales de "conceptos" y "procesos" en el pensamiento computacional.

La figura 3 muestra, en la parte izquierda al docente con sus alumnos impartiendo clase en modo mixto (presencial y plataforma online- Educando en línea ), y en la parte derecha la herramienta barra de progreso de los alumnos.
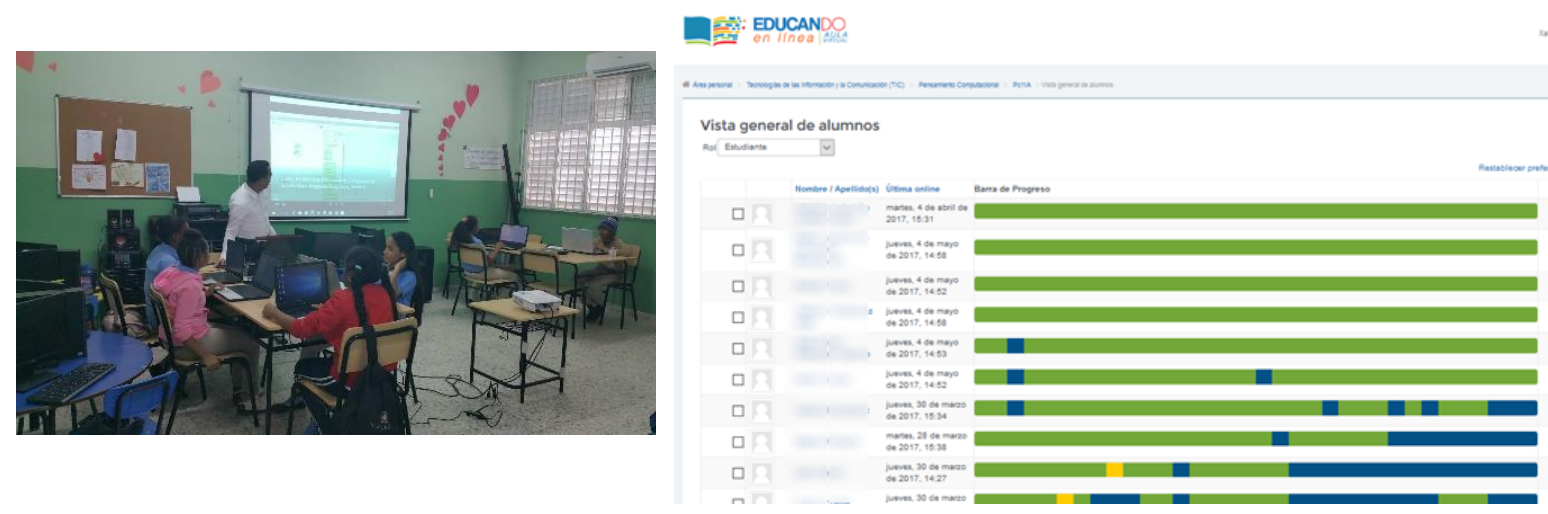

Figura 3.- Docente impartiendo clase en formato mixto y herramienta para el seguimiento del progreso de los alumnos.

El curso PC-01 ha sido diseñado y creado por un equipo docente e investigador de la Universidad del País Vasco - Euskal Herriko Unibertsitatea al que pertenece uno de los autores del artículo. El curso PC-01 se ha desplegado en colaboración con la Dirección General Informática Educativa del Ministerio de Educación (MINERD) de la República Dominicana en más de 30 escuelas de todo el país.

El equipo docente del curso ha colaborado y asistido a los maestros a través de reuniones virtuales (Skype y Blackboard Collaborate). Antes de comenzar el curso los docentes reciben capacitación sobre la metodología y contenido del curso. Para ayudar completamente al maestro local, se ha creado una guía completa que describe la estructura y el funcionamiento de las partes principales del curso. Esta documentación incluye una descripción de los temas estudiados en el curso, los recursos pedagógicos disponibles, cómo se organiza el curso en sesiones modulares y cómo estas sesiones deben ser presentadas a los estudiantes. En las áreas de evaluación, tanto la evaluación individual como la evaluación entre pares o iguales, la guía proporciona directrices para los docentes y los estudiantes sobre el uso óptimo de estos recursos. Debido a que la evaluación de TEC es en general una nueva herramienta para la mayoría de las instituciones, se realiza con énfasis especial la descripción de las tres fases de evaluación, los criterios para calificar el trabajo presentado y cómo se obtienen las calificaciones finales. Por último, se adjunta un índice completo que incluye los recursos disponibles (tutoriales en video, proyectos prácticos, auto-test, test y las soluciones para los proyectos TEC).

Integración de Pensamiento Computacional en Educación Básica. Dos Experiencias Pedagógicas de Aprendizaje Colaborativo online. Xabier Basogain y María Elena Olmedo

Página 11 de 21 


\section{DISCUSIÓN Y CONCLUSIONES}

El Pensamiento computacional es una habilidad que ha tomado relevancia en el mundo digital actual. Uruguay está a la vanguardia junto a países como España, Alemania, Estonia, Reino Unido y Finlandia, entre otros, y en la región es referencia para países vecinos como Paraguay y Argentina, y otros países de Latinoamérica que están comenzando a introducir la programación en sus sistemas educativos. Los niños aprenden razonamiento lógico, pensamiento algorítmico y técnicas de resolución de problemas, así como a expresar sus ideas, creatividad y habilidades de diseño. En algunos países de Europa la propuesta integra también las áreas STEAM (Ciencia, Tecnología, Ingeniería, Arte y Matemática, por su sigla en inglés), aprendizaje basado en proyectos y trabajo con material concreto.

\section{Resultados}

Las dos experiencias descritas en los apartados anteriores se han realizado desde iniciativas institucionales que buscan una aproximación a la incorporación del PC en las aulas de sus centros escolares. El carácter de las mismas no es un estudio de investigación sino ser unas propuestas específicas y realizables. Estas experiencias han reportado conocimiento e información cuantitativa y cualitativa. En los siguientes apartados detallamos algunos de estos resultados.

\section{El Portfolio}

La construcción del portfolio del estudiante en la experiencia curso PC-01 se implementa con la acumulación de proyectos creados como parte de los TEC. Estos proyectos documentan las habilidades adquiridas por los estudiantes, incluyendo los paradigmas de programación y las ideas centrales correspondientes del Pensamiento Computacional.

La tabla 3 enumera la colección de 10 proyectos TEC implementados en el mencionado curso PC-01 y la idea principal de cada proyecto (Basogain et al., 2017).

Tabla 3. Lista de Proyectos TEC.

\begin{tabular}{|c|c|c|}
\hline № & Proyecto & Idea principal PC \\
\hline TEC 1 & $\begin{array}{l}\text { Diseño de un proyecto para dibujar tres } \\
\text { triángulos en el escenario. }\end{array}$ & $\begin{array}{l}\text { Secuencia de instrucciones y control de } \\
\text { repetición. }\end{array}$ \\
\hline TEC 2 & $\begin{array}{l}\text { Diseño de un proyecto donde el } \\
\text { protagonista narra una breve historia } \\
\text { personal. }\end{array}$ & $\begin{array}{l}\text { Uso de disfraces y mensajes de texto } \\
\text { asociados a un sprite (objeto). }\end{array}$ \\
\hline TEC 3 & $\begin{array}{l}\text { Diseño de un proyecto con } 4 \text { protagonistas } \\
\text { tocando diferentes sonidos. }\end{array}$ & $\begin{array}{l}\text { Uso de múltiples sprites y reproducción de } \\
\text { procedimientos de sonido. }\end{array}$ \\
\hline TEC 4 & $\begin{array}{l}\text { Diseño de un proyecto donde el } \\
\text { protagonista dibuja una escena de } \\
\text { imágenes de arte creativo con líneas y } \\
\text { curvas de múltiples colores. }\end{array}$ & $\begin{array}{l}\text { Control del recurso del lápiz del sprite, } \\
\text { incluyendo el color y la posición. }\end{array}$ \\
\hline TEC 5 & $\begin{array}{l}\text { Diseño de un proyecto para permitir al } \\
\text { usuario dibujar en el escenario con un } \\
\text { bolígrafo de múltiples colores. }\end{array}$ & $\begin{array}{l}\text { Uso de eventos y controladores de eventos con } \\
\text { bucles de control simples. }\end{array}$ \\
\hline TEC 6 & $\begin{array}{l}\text { Diseño de un proyecto para implementar un } \\
\text { reloj con una aguja en movimiento asociada } \\
\text { a múltiples efectos sonoros y gráficos. }\end{array}$ & $\begin{array}{l}\text { Estructuras de control e introducción a la } \\
\text { programación multihilo. }\end{array}$ \\
\hline TEC 7 & $\begin{array}{l}\text { Diseño de un proyecto con los sprites bola, } \\
\text { escarabajo, cangrejo y pelota de playa } \\
\text { interactuando entre sí utilizando sensores } \\
\text { del entorno. }\end{array}$ & $\begin{array}{l}\text { Sensores que informan sobre el entorno (teclas } \\
\text { pulsadas, pregunta sobre los valores } \\
\text { almacenados en el escenario, etc.). }\end{array}$ \\
\hline
\end{tabular}

Integración de Pensamiento Computacional en Educación Básica. Dos Experiencias Pedagógicas de Aprendizaje Colaborativo online. Xabier Basogain y María Elena Olmedo

Página 12 de 21 


\begin{tabular}{|l|l|l|}
\hline TEC 8 & $\begin{array}{l}\text { Diseño de un proyecto donde un pingüino } \\
\text { exhibe talentos matemáticos. }\end{array}$ & $\begin{array}{l}\text { Uso de operadores numéricos y de texto, } \\
\text { aritmética, aleatoria, concatenación. }\end{array}$ \\
\hline TEC 9 & $\begin{array}{l}\text { Diseño de un proyecto para controlar la } \\
\text { velocidad y el color de una nave espacial } \\
\text { mediante el uso de variables. }\end{array}$ & $\begin{array}{l}\text { Manipulación sencilla de datos (ajuste y } \\
\text { cambio de contenido de variables). }\end{array}$ \\
\hline TEC 10 & $\begin{array}{l}\text { Diseño de un proyecto para la creación de } \\
\text { nuevos bloques de programación definidos } \\
\text { por el usuario. Los nuevos bloques } \\
\text { permitirán el dibujo complejo de patrones } \\
\text { geométricos con parámetros variables. }\end{array}$ & $\begin{array}{l}\text { Bloques diseñados por el usuario Más Bloque: } \\
\text { floques personalizados para abstraer la } \\
\text { programación sea una tarea modular. }\end{array}$ \\
\hline
\end{tabular}

\section{Analítica de Aprendizaje}

Una de las ventajas de EVA o de la plataforma de aprendizaje es la explotación de datos del aprendizaje (analytic learning) (Singh, 2015).

En particular hemos analizado del curso PC-01 los siguientes ítems o variables: 1) los datos correspondientes al número de intentos en realizar los Autotest y Test, y 2) los datos correspondientes a la calificación obtenida en los Autotest y Test.

El estudio se ha realizado en dos aulas particulares, aula $A$ y aula B (denomiados PC10 y PC12 de la plataforma http://aula.educando.edu.do) correspondientes a dos escuelas de las localidades Las Matas de Farfán y San Pedro de Macorís de la República Dominicana. Los valores promedios numéricos de las dos variables analizadas para las aulas $A$ y $B$ se muestran en la siguiente tabla 4:

Tabla 4. Promedios de las Calificaciones e Intentos de las aulas A y B

\begin{tabular}{|l|c|c|c|c|}
\cline { 2 - 5 } \multicolumn{1}{c|}{} & \multicolumn{2}{c|}{ Autotest } & \multicolumn{2}{c|}{ Test } \\
\hline & Calificación & Intentos & Calificación & Intentos \\
\hline PC12A & 54,75 & 21,75 & 62,75 & 14,13 \\
\hline PC10A & 85,90 & 17,30 & 94,40 & 12,50 \\
\hline
\end{tabular}

La figura 4 muestra los resultados obtenidos en cada una de las 10 sesiones del curso $\mathrm{PC}$ en las aulas $\mathrm{A}$ y $\mathrm{B}$ en el número de intentos del auto-test y test, así como las calificaciones obtenidas respectivamente.

Intentos en las aulas A y B

$\% n^{\circ}$ de intentos en Test y Autotest

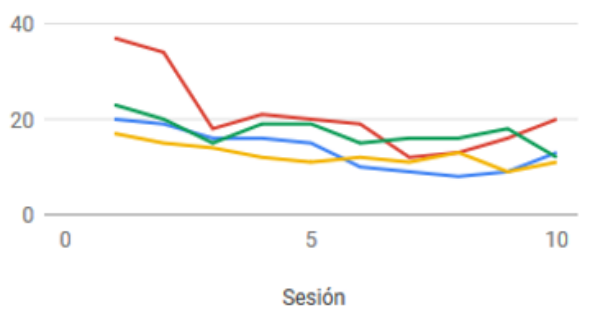

Calificaciones en las aulas A and B $\%$ notas obtenidas en Test y Autotest

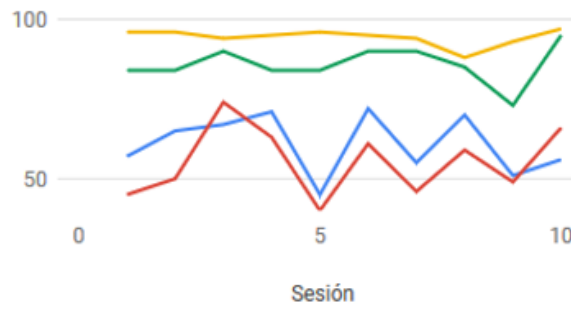

Figura 4.- Intentos en realizar los autotest y test a lo largo de las 10 sesiones/temas del curso PC-01. 
La parte izquierda de figura 4 muestra el número total de intentos de auto-test y test en estas aulas. Se puede observar la tendencia descendente general a lo largo de las diez sesiones semanales del curso. Esto indica que los estudiantes inicialmente optan por practicar por períodos más largos de tiempo cuando la materia a aprender es todavía nueva y desconocida. A medida que avanza el curso, los estudiantes experimentan una mayor confianza en sus habilidades y conocimientos, y por lo tanto tiene menos necesidad de realizar intentos adicionales. Hay una diferencia visible entre ambas aulas, pero la diferencia es más cuantitativa que cualitativa.

La parte derecha de la figura 4 ilustra las calificaciones obtenidas en los auto-test y en los test. Se observa una tendencia general en la que se obtiene mejores calificaciones en los test que en los auto-test a lo largo de las 10 sesiones semanales del curso. Esto indica que los estudiantes han ido reforzando gradualmente sus conocimientos sobre los fundamentos básicos, a medida que van acumulando experiencia y tiempo realizando las evaluaciones y dedicando esfuerzo en los diseños de los proyectos.

De estas gráficas se pueden inferir otros resultados de gran interés para el docente del aula como para los centros educativos o la administración educativa del país. Entre otros resultados destacamos los siguientes: a) diferencias de hábito a la hora de practicar con los autotest y test (una escuela hace más que la otra), y b) identificación de una sesión con malos resultados (como se observa en la figura, la sesión 5 del aula A).

\section{Discusión y Líneas Futuras}

Se están produciendo numerosos cambios e innovaciones en aulas, escuelas y sistemas. Muchos de ellos son motivadores, pero como sucede en los albores de toda innovación, suponen un grado de incertidumbre en cuanto a los resultados que ellas arrojarán en el corto, mediano y largo plazo. No sabemos tampoco cuál será el grado de injerencia en la relación docente-alumno; ni el impacto que estos tendrán en los sistemas educativos correspondientes. Lo que sí sabemos es que estamos a las puertas de un proceso de cambios en los modos enseñar y aprender originado en la necesidad de reajustes y modificaciones en la educación actual.

En esta sección nos centramos en indagar en la búsqueda de percepciones que puedan llevar a concluir que estas nuevas pedagogías tienen un impacto positivo en el salón de clases basándonos en los resultados de las experiencias relatadas, pero también con la certeza de que nos encontramos en la primera etapa de un camino que recién empieza y en cuyo final hemos depositado fundadas expectativas.

\section{Los cambios en las pedagogías puestos en juego en las clases de Pensamiento Computacional.}

En el dictado de las clases de PC se suceden imprevistos de orden pedagógico que han dejado bien en claro que son necesarias otras pedagogías mucho más complejas, efectivas y pragmáticas que las tradicionales. Además, se suman las tecnologías y el contacto con el mundo que éstas conllevan, alcanzando lo que Fullan llama "aprendizaje profundo" (Fullan \& Langworthy, 2014).

Es imperativo un aprendizaje que supere el mero dominio de los contenidos propuestos por el docente; porque la identificación y resolución del Problema Situado llevan a los estudiantes a aprender dentro y fuera de la escuela, durante toda la vida y para todos los aspectos de la vida cotidiana. Se aprende diferente con cada Problema Situado en la 
necesidad de que sean los estudiantes quienes lo aborden para poder comprenderlo, interiorizarlo, asumirlo. Sólo así tiene sentido pensar alternativas de solución.

En relación a la estrategia pedagógica en el momento de poner en valor lo realizado, no resulta sencillo indicar un momento preciso en el cual puede decirse que se ha logrado el fin buscado. Cada clase se piensa más como un proceso a ser recorrido que tiene por hilo conductor el Problema elegido. Cada semana los alumnos logran desarrollar conocimientos relacionados a la programación, direccionar su aprendizaje a través de la elección del Problema que afecta a su comunidad, determinando y perseverando en los pasos para su solución. Este enfoque pedagógico está cada vez más presente en el trabajo docente, a través de proyectos que orientan los aprendizajes hacia el desarrollo del carácter, la creatividad, el pensamiento crítico, la colaboración, la comunicación y la ciudadanía.

Con el impulso de nuevas tecnologías disponibles para la educación, las formas de enseñar y de aprender se están transformando. La observación del entorno y los problemas de la vida real son los disparadores de experiencias de aprendizaje a través del hacer. Y la docencia es hoy, más que nunca, una actividad colectiva.

\section{Docencia apoyada con Entorno Virtual de Aprendizaje (EVA)}

Uno de los cambios fundamentales en la educación con la asistencia de los EVA es la creación del modo colaborativo de educación, donde los estudiantes reciben sus conocimientos tanto del docente local, presente en el aula, como del conocimiento multimedia basado en el EVA. Por ejemplo, para ofrecer cursos de Pensamiento Computacional en una institución, no es necesario que el docente local del aula sea un experto en el campo. Lo importante es que el maestro tenga conocimiento del curso PC implementado en el EVA. El contenido del curso, evaluaciones, proyectos y otras tareas académicas son proporcionados por lo que llamamos el "docente remoto digital". El docente local apoyado por el EVA implementa las tareas de líder del aula, motivador y director de las actividades en todo momento en el aula.

La plataforma EVA del curso permite al estudiante, además de desempeñar el papel de evaluador, colaborar con los compañeros dando y recibiendo comentarios, indicando errores, soluciones y posibles mejoras. Además de estar expuestos a otros proyectos (por ejemplo de Scratch), estas actividades abren al estudiante la oportunidad de aprender e inspirarse en nuevas soluciones para problemas futuros (Lu \& Law, 2012).

La experiencia de los estudiantes en estos cursos híbridos les permite también familiarizarse con las herramientas y metodologías de los EVA. Como estudiantes de un sistema EVA han experimentado diferentes aspectos de esta forma de aprendizaje: contenidos en formato multimedia, mecanismos de colaboración, mecanismos de autoevaluación y evaluación, progreso en sus conocimientos y calificaciones.

Los cursos ofrecen una insignia que es un premio o medalla para reconocer sus logros y proporcionar a los estudiantes una recompensa que reconozca su aprovechamiento en el Pensamiento Computacional (Seliskar, 2014).

En un futuro a medio plazo estos estudiantes verán su propio progreso de conocimiento a través del uso de herramientas y servicios de plataformas educativas en línea que ofrecen cursos masivos de MOOC (siglas en inglés de Massive Online Open Courses- Cursos Online Masivos y Abiertos). El aprendizaje a lo largo de toda la vida exigirá a los estudiantes el uso

Integración de Pensamiento Computacional en Educación Básica. Dos Experiencias Pedagógicas de Aprendizaje Colaborativo online. Xabier Basogain y María Elena Olmedo

Página 15 de 21 
de plataformas EVA similares al entorno experimentado por el estudiante en este tipo de cursos (Attwell \& Hughes, 2010).

\section{Próximos pasos: Educación STEAM y ecosistemas computacionales}

Los sistemas educativos de todo el mundo se han embarcado durante la última década en un conjunto de proyectos paradigmáticos ambiciosos que se pueden agrupar bajo los nombres generales de STEM y STEAM. Dos ideas fundamentales motivan estos proyectos. La primera idea es que los ciudadanos de la sociedad moderna deben tener una educación sólida en las áreas de STEM (en inglés Ciencia, Tecnología, Ingeniería y Matemáticas) o STEAM (agregando las Artes y las Humanidades). La segunda idea es que estas materias necesitan ser enseñadas y aprendidas no como temas separados y aislados, sino como componentes integrales de una entidad superior.

Como se esperaba en cualquier proyecto a gran escala, particularmente cuando incluye diversos grupos, como la academia, la industria y los responsables políticos nacionales, las fases iniciales de los proyectos revelan conflictos fundamentales (EU STEM, 2019). Estos emanan de los supuestos que cada comunidad participante trae a la mesa cómo entienden los objetivos y los mejores caminos para alcanzarlos. Existen tres obstáculos fundamentales que actualmente impiden el desarrollo con éxito de proyectos STEAM a gran escala.

Para abordar estos obstáculos definimos una ontología de estos proyectos, denominada STEAM Computacional (Olabe, Basogain \& Olabe, 2019). Esta ontología se usa para describir un proyecto STEAM en términos computacionales. Incluye los objetivos establecidos por la industria, el contenido destinado a ser incluido en el plan de estudios, la metodología para la enseñanza y el aprendizaje y las herramientas de trabajo que los ciudadanos utilizarán en la sociedad moderna.

En este contexto destacamos que los nuevos planes de estudio de educación primaria y secundaria en las áreas de STEAM deberán incluir nuevas materias, como el cálculo discreto, la geometría vectorial diferencial, la computación probabilística y la cibernética como nuevo contenido matemático en el paradigma de STEAM computacional (Papert, 1996; Abelson \& DiSessa, 1986; Kay, 1991; Papert, 2005) a pesar de que estos temas no forman parte del plan de estudios escolar actual. Además, la computación como lenguaje generativo de acción introduce la idea fundamental de crear sistemas y procesos como medio para avanzar en el conocimiento, proporcionar servicios y resolver problemas.

El ordenador es el candidato ideal para la creación de entornos computacionales donde se lleva a cabo el aprendizaje. El conocimiento en estos entornos debe definirse explícitamente y describirse formalmente. Los sistemas complejos requieren una matemática generativa que implemente una actividad computacional y también requieren un entorno computacional, el ordenador, donde se produce la experimentación y el aprendizaje.

La figura 5 ilustra un ecosistema computacional realizado con el entorno de programación Snap para la creación de los patrones formados a medida que se crean las semillas en un girasol ${ }^{4}$. La imagen final muestra los diferentes conjuntos de diagonales curvas que emanan del centro de la flor. Los tres conjuntos principales de diagonales curvas confirman su regla de los números de Fibonacci: 34, 55 y 89.

\footnotetext{
${ }^{4}$ https://snap.berkeley.edu/project?user=steam-euskadi\&project=EHU-SP2-01_Girasol\%20de\%20Fibonacci Integración de Pensamiento Computacional en Educación Básica. Dos Experiencias Pedagógicas de Aprendizaje Colaborativo online. Xabier Basogain y María Elena Olmedo

Página 16 de 21
} 


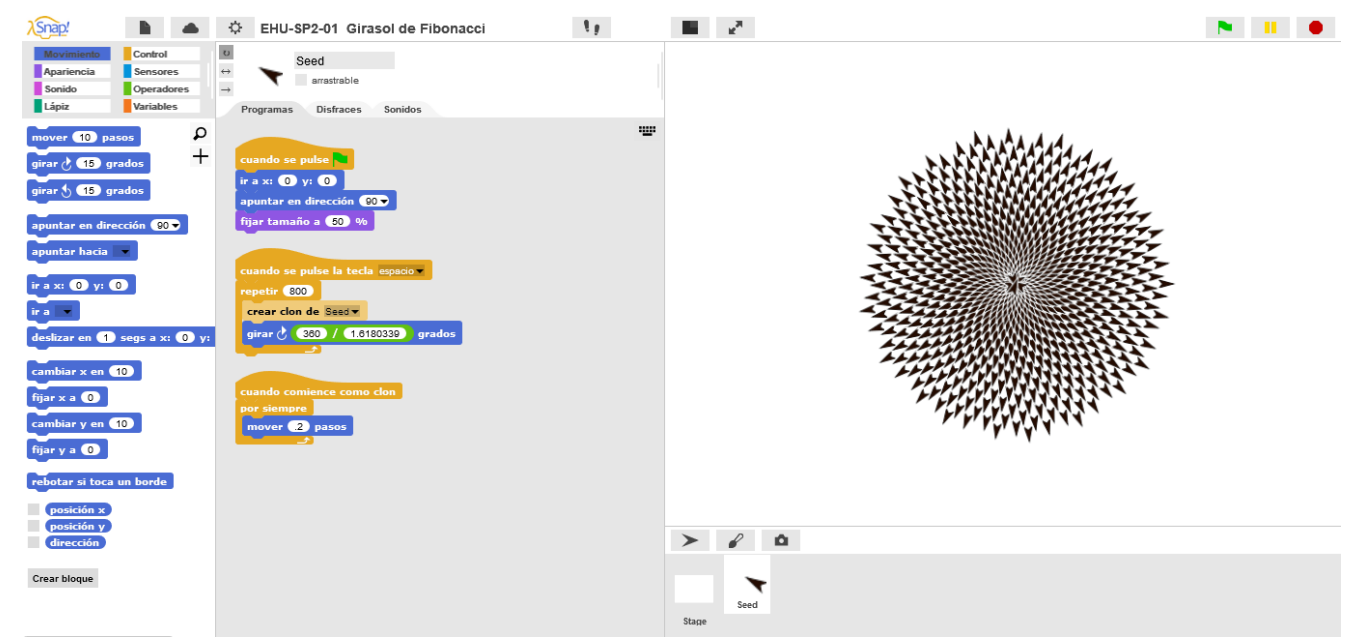

Figura 5.- Ecosistema computacional para la ilustración de patrones de girasoles y su relación con la proporción áurea y los números de Fibonacci realizado en el entorno de programación Snap

Este ejemplo de geometría vectorial diferencial muestra la paradoja de la complejidad donde con solo dos reglas básicas se pueden crear sistemas de aparente gran complejidad. El ecosistema computacional crea 800 semillas idénticas: todas se crean en el centro de la flor. Después de crear cada semilla, el generador de semillas la gira una fracción de un círculo. El giro está determinado por la proporción áurea: 1.6180339. Una vez que se ha creado una semilla, sigue un camino rectilíneo externo a un ritmo muy lento. A medida que se crean las semillas, la flor sigue creciendo en tamaño y los patrones comienzan a ser reconocibles.

La tecnología educativa juega un papel fundamental en el nuevo paradigma de enseñanza y aprendizaje. Los entornos de programación como Scratch, Snap y otros son complementados por otras aplicaciones informáticas que abren las posibilidades de aumentar el conocimiento del mundo que nos rodea (Aparicio y Ostos, 2019). Por ejemplo, ¿es posible enseñar a los niños nuevos conceptos como Machine Learning o Aprendizaje Automático? Los asistentes personales inteligentes nos acompañan en el día a día en nuestros dispositivos móviles. Pronto otros sistemas de aprendizaje automático nos van asistir conduciendo nuestros automóviles, ayudarán a los médicos a diagnosticar y tratar nuestras enfermedades, o nos propondrán planes lúdicos para nuestras vacaciones.

La introducción del Machine Learning a los niños (Lane, 2019) es una forma de que los niños empiecen a conocer cómo funciona el mundo. La mejor manera de comprender las capacidades e implicaciones del aprendizaje automático, es usar esta tecnología para poder construir algo ellos mismos. Un ejemplo es el software Machine Learning for Kids (Aprendizaje Automático para Niños, 2019). Se trata de un entorno de aprendizaje guiado para entrenar modelos de aprendizaje automático capaces de identificar textos, números o imágenes y que se utiliza con Scratch. Los niños aprenden cómo se entrenan los sistemas de aprendizaje automáticos, cómo se usan y algunas de las implicaciones en el mundo real de las aplicaciones de la Inteligencia Artificial. Ya se están creando materiales didácticos accesibles a la comunidad educativa para introducir Machine Learning con Scratch en las escuelas (García, León, González and Robles, 2019). 


\section{Conclusiones}

El Pensamiento Computacional es una materia que se está incorporando en la educación básica de todos sistemas escolares del mundo. Existen muchas iniciativas con diferentes estrategias y métodos que buscan integrar en el aula el PC. En este artículo hemos descrito dos experiencias realizadas en dos países de Latinoamérica que ponen en valor el aprendizaje colaborativo apoyado en las tecnologías de la información y en el apoyo de expertos docentes en la materia.

El aprendizaje colaborativo se desarrolla entre el docente del aula y los estudiantes con la asistencia presencial telemática del docente remoto $\mathrm{y} / \mathrm{o}$ del docente remoto digital (integrado en la plataforma EVA). Gracias a esta colaboración múltiple el proceso de aprendizaje se desarrolla con éxito en el aula donde un docente de aula es capaz de introducir el PC a sus estudiantes y realizar un seguimiento formativo de todos los estudiantes, y a su vez, los estudiantes son protagonistas de su propio aprendizaje desarrollando tanto competencias básicas-transversales como competencias específicas del Pensamiento Computacional.

Presentación del artículo: 28 de febrero de 2020 Fecha de aprobación: 2 de marzo de 2020 Fecha de publicación: 30 de abril de 2020

Basogain, X. y Olmedo, M.E. (2020). Integración de Pensamiento Computacional en Educación Básica. Dos Experiencias Pedagógica de Aprendizaje Colaborativo online. RED Revista de Educación a Distancia, 20(63). DOI: http://dx.doi.org/10.6018/red.409481

\section{Financiación}

Esta investigación no ha recibido ninguna subvención específica de los organismos de financiación en los sectores públicos, comerciales o sin fines de lucro.

\section{REFERENCIAS}

Abelson, H. \& DiSessa, A. (1986). Turtle geometry: The computer as a medium for exploring mathematics. MIT press.

Aparicio, O. Y. \& Ostos, O. L. (Eds.). (2019) (En prensa). Innovación Educativa y Gestión del Conocimiento, Bogotá: Universidad Santo Tomás

Aprendizaje Automático para Niños (2019). Machine Learning for Kids. Consultado el 28/2/2020 en https://machinelearningforkids.co.uk/\#!/about

Attwell, G. \& Hughes, J. (2010). Pedagogic approaches to using technology for learning: Literature review.

Basogain Olabe, X., Olabe Basogain, M. Á. \& Olabe Basogain, J. C. (2015). Pensamiento Computacional a través de la Programación: Paradigma de Aprendizaje. RED Revista De Educación a Distancia, 46(6). DOI: https://doi.org/10.6018/red/46/6 
Basogain, X., Olabe, M. A., Olabe, J. C., Ramírez, R., Del Rosario, M. and Garcia, J. (2016). PC-01: Introduction to Computational Thinking. Educational Technology in Primary and Secondary Education. 2016 International Symposium on Computers in Education (SIIE). pp. 1-5. DOI: http://dx.doi.org/10.1109/SIIE.2016.7751816

Basogain, X., Olabe, M. Olabe, J.C., Rico, M., Rodríguez, L., \& Amortegui, M. (2017). Pensamiento computacional en las escuelas de Colombia: colaboración internacional de innovación en la educación. Virtual Educa 2017, Bogotá, Colombia. Consultado el 28/2/2020 en http://recursos.portaleducoas.org/publicaciones/pensamientocomputacional-en-las-escuelas-de-colombia-colaboraci-n-internacional-de

Basogain, X., Olabe, M. A., Olabe, J. C. Rico, M. (2018). Computational Thinking in PreUniversity Blended Learning Classrooms. Journal Computers in Human Behavior, 80 (March), 412-419. DOI: https://doi.org/10.1016/j.chb.2017.04.058

College Board (2016). AP Computer Science Principles. Course and Exam Description. Consultado el 28/2/2020 en: https://securemedia.collegeboard.org/digitalServices/pdf/ap/ap-computer-science-principlescourse-and-exam-description.pdf

CREA2 (2018). Plataforma de Aprendizaje del Equipo de laboratorios digitales. Organización de la Videoconferencia. Plataforma CREA2. Plan Ceibal. Uruguay

Cyranek, G. (2009). En el camino del plan CEIBAL: referencias para padres y educadores. UNESCO Office Montevideo and Regional Bureau for Science in Latin America and the Caribbean. Montevideo, Uruguay.

Decreto 236/2015 (2016). Decreto 236/2015, de 22 de diciembre, por el que se establece el currículo de la Educación Básica y se implanta en la Comunidad Autónoma del País Vasco (2016). BOVP, Boletín Oficial del País Vasco, № 9, de 15-01-2016. Consultado el 28/2/2020 en https://www.euskadi.eus/y22bopv/es/bopv2/datos/2016/01/1600141a.pdf

EU STEM Coalition (2019). Strengthening STEAM education in the EU. Dictamen del Comité Europeo de las Regiones - Reforzar la educación en ciencia, tecnología, ingeniería (artes) y matemáticas [CTI(A)M] en la Unión Europea, 2019. Consultado el 28/2/2020 en https://www.stemcoalition.eu/publications/strengthening-steam-education-eu

Fullan, M. \& Langworthy, M. (2013). Towards a new end: New pedagogies for deep learning. Collaborative Impact Publisher.

Fullan, M. \& Langworthy, M. (2014). Una rica veta Cómo las nuevas pedagogías logran el aprendizaje en profundidad. Stanford (California-EE.UU.): Pearson. Recuperado de https://www.pearson.com/content/dam/one-dot-com/one-dot-com/global/Files/aboutpearson/innovation/open-ideas/ARichSeamSpanish.pdf

García, J. D. R., León, J. M., González M. R. and Robles, G. (2019) Developing Computational Thinking at School with Machine Learning: An exploration, 2019 International Symposium on Computers in Education (SIIE), Tomar, Portugal, 2019, pp. 1-6. doi: 10.1109/SIIE48397.2019.8970124 
Gerosa, A., Koleszar, V., Carboni, A. y Gómez, L. (2019). Desafiando al Pensamiento Computacional. Consultado el 28/2/2020 en http://www.cicea.ei.udelar.edu.uy/pensamientocomputacional/

Kay, A. C. (1991). "Computers, networks and education." Scientific American 265, no. 3 (1991): 138-149.

Lane, D. (2019). Introducing Machine Learning to kids. Consultado el 28/2/2020 en https://dalelane.co.uk/blog/?p=3513

Lévy, P. (2004). Inteligencia colectiva: por una antropología del ciberespacio. Consultado el $28 / 2 / 2020$

en: http://inteligenciacolectiva.bvsalud.org/public/documents/pdf/es/inteligenciaColectiva. pdf

LOMCE (2013). Ley Orgánica 8/2013, de 9 de diciembre, para la mejora de la calidad educativa (LOMCE). BOE, 295, de 10 de diciembre. Consultado el 28/2/2020 en https://www.boe.es/boe/dias/2013/12/10/pdfs/BOE-A-2013-12886.pdf

Lu, J. \& Law, N. W. Y. (2012). Understanding collaborative learning behavior from Moodle log data. Interactive Learning Environments, 20(5), 451-466.

Maloney, J., Resnick, M., Rusk, N., Silverman, B., \& Eastmond, E. (2010). The Scratch Programming Language and Environment. ACM Transactions on Computing Education, 10 (4), 15 pp. DOI: https://doi.org/10.1145/1868358.1868363

Ministerio de Educación de la República Argentina (2017)- Abordaje y resolución de Situaciones problemáticas- Documento de Acompañamiento № 13 . Programa Nuestra Escuela.

Olabe, J.C., Basogain, X., Olabe, M., Maíz, I. \& Castaño, C. (2014). Solving math and science problems in the real world with a computational mind. Journal of New Approaches in Educational Research, 3(2), 75-82. DOI: http://dx.doi.org/10.7821/naer.3.2.75-82

Olabe, J. C., Basogain, X. \& Olabe, M. A. (2019). Chapter 1. An Ontology of Computational STEAM: The Role of Educational Technology En M. A. Clausen (Ed.), A Closer Look at Educational Technology. Series: Technology in a Globalizing World (pp. 1-24). New York, Nova Science Publishers.

Olmedo Parco, M.E. (2013). La pedagogía frente a la pantalla. El Programa JVG una ventana al mundo. La Rioja. Argentina.

Papert, S. (1996). An exploration in the space of mathematics educations. International Journal of Computers for Mathematical Learning, 1(1), 95-123.

Papert, S. (2005). Teaching children thinking. Contemporary issues in technology and teacher education, 5(3), 353-365. DOI: https://doi.org/10.1007/BF00191473

Scratch (2019). Lifelong Kindergarten Group at the MIT Media Lab. Consultado el 28/2/2020 en http://scratch.mit.edu/

Seliskar, H. V. (2014). Using Badges in the Classroom to Motivate Learning. Faculty Focus. Magna Publications. Consultado el 28/2/2020 en 
http://www.facultyfocus.com/articles/teaching-with-technology-articles/using-badgesclassroom-motivate-learning/

Singh, J. (2015). Learning Analytics tools available in Moodle. Consultado el 28/2/2020 en http://www.moodleworld.com/learning-analytics-tools-available-in-moodlemoodleresearch-moodleworld/

Snap (2019). Snap! Build Your Own Blocks. University of California at Berkeley. Consultado el 28/2/2020 en https://snap.berkeley.edu/

Utting, I., Cooper, S., Kolling, M., Maloney, J. \& Resnick, M. (2010). Alice, Greenfoot and Scratch-A discussion. ACM Transactions on Computing Education, 10(4). DOI: https://doi.org/10.1145/1868358.1868364

Wing, J. (2006). Computational Thinking. View Point. Comunication of ACM. Vol. 49, No. 3. Pág. $35 . \quad$ Consultado el $28 / 2 / 2020$ en http://www.cs.cmu.edu/afs/cs/usr/wing/www/publications/Wing06.pdf 